Research Article

\title{
Colorimetric Detection of Ammonia Using Synthesized Silver Nanoparticles from Durian Fruit Shell
}

\author{
Eman Alzahrani (iD \\ Department of Chemistry, College of Science, Taif University, P.O. Box 11099, Taif 21944, Saudi Arabia \\ Correspondence should be addressed to Eman Alzahrani; em-s-z@hotmail.com
}

Received 10 August 2020; Revised 21 September 2020; Accepted 22 September 2020; Published 20 October 2020

Academic Editor: Patricia E. Allegretti

Copyright (C) 2020 Eman Alzahrani. This is an open access article distributed under the Creative Commons Attribution License, which permits unrestricted use, distribution, and reproduction in any medium, provided the original work is properly cited.

\begin{abstract}
There has been increased interest in the production of nanoparticles (NP) through green chemistry. This article used durian fruit shell aqueous solution that acts as a reductive preparation of silver NPs. The silver nanoparticles have a size of approximately $25 \mathrm{~nm}$. The NP size uniformity was determined by the SEM and TEM analysis. X-ray diffraction technique was used to characterize crystalline silver nanoparticles face-centered cubic structure. XPS spectrum showed distinct silver peaks on the nanoparticles' surface. An optical method that was based on surface plasmon resonance (SPR) was used to perform the green Ag NPs aqueous ammonia sensing study. Optical measurement facilitated the ammonia sensing study of Ag NPs that had been prepared. The study also investigated the performance of the optical sensor, thus adding validity to the study. Also, the research sought to determine how the concentration of ammonia in ammonia sensing affects the Ag NPs that had been obtained. The study observed a linear relationship with $R^{2}$ as the correlation factor which was equal to 0.9831 . This was observed from the ammonia concentration plot versus absorption ratio that suggested that there was a linear increase in absorption ratio with increase in ammonia concentration. The study significance is that the room temperature optical ammonia sensor can be used in future for medical diagnosis in the detection of low levels of ammonia in biological fluid like sweat, cerebrospinal fluid, saliva, plasma, or biological samples. This enhances the application of the technique in human biomedical applications.
\end{abstract}

\section{Introduction}

Ammonia is produced by the process of nitrogen fixation, decomposition of organic compounds, and natural exchange of atmospheric gases [1-6]. The gas has broad applications including the pharmaceutical industry, manufacture of fertilizers, production of explosives, and manufacture of plastics. Ammonia is also used as a synthetic paper and in the manufacture and production of paper and large-scale food refrigeration [7-9]. Ammonia also has detrimental effects on human health as it creates eye and skin irritation. High ammonia concentration has been implicated in lung disorders and permanent blindness. The gas is hazardous to crustaceans, an aquatic species, even in minimal concentrations. The gas cannot be converted to compounds that are less toxic. The presence of ammonia in the body of humans could be an indication of various diseases and disorders. Some of the diseases and disorders that can be detected by the presence of ammonia include kidney and liver collapse, diabetes, ulcers, bacterial infections of the gut, and cancer [10-14].

It is important to find effective ways of detecting the presence of ammonia even at low concentration because of its toxic nature [15-17]. Various strategies of detecting the presence of ammonia have been described on the basis of Nessler reaction and luminol-hypochlorite reaction $[18,19]$. There are, however, several drawbacks that affect the effective detection of dissolved ammonia. Additional equipment is required in the ammonia detection process and the related reactions produce toxic chemicals.

Silver NPs has gained immense relevance in the recent past in the detection of various chemicals through colorimetric sensing because of its effective optical features called surface plasmon resonance (SPR) [20-23]. The latter is an optical phenomenon that is seen when the surface conducting electrons of Ag NPs are excited by electromagnetic 
radiation leading to incoherent resonance oscillation within the particle $[24,25]$. When the environment is manipulated through a change in reflective index of the medium surrounding the particles and the metal surface molecules adsorption, absorbance change in near-infrared and visible wavelength regions is seen in UV-vis spectrometry and is also visible by naked eyes in certain situations [26, 27].

The colorimetric sensing of Ag NPs has been reported by various researchers. Silver NPs were synthesized via peach gum polysaccharides by Yang et al. [28]. The NPs that were obtained from the procedure exhibited color changes from brown to colorless for a detection limit of $0.035 \mathrm{mM}$ for hydrogen peroxide. The Ag NPs were synthesized via Ficus amplissima leaf extract under direct sunlight by Manivel and Ilanchelian [29]. The synthesized NPs were used for sensing mercury ion. In the presence of $\mathrm{Hg}^{2+}$. the yellow silver NPS colloids changed color to become colorless with varying $\mathrm{pH}$ levels from 3.2 to 8.5. Silver NPs were prepared using soluble starch and formamidine sulfinic acid by Song et al. [30]. The $\mathrm{Ag}$ NPs that were produced exhibited calorimetric sensing for various metal ions. There was a distinct color change for $\mathrm{Cr}^{3+}$ from yellow to orange, yellow to colorless for $\mathrm{Fe}^{3+}$ and $\mathrm{Hg}^{2+}$, and yellow to pink for $\mathrm{Al}^{3+}$. Various studies on ammonia detection have been conducted in the recent past. One example is Dubas and Pimpan's [31] study where ammonia sensing of Ag NPs was analyzed and stabilized by poly(methacrylic acid) and sodium borohydride. There was a distinct change of color from purple to yellow following the addition of a solution of ammonia at a limit of detection of $5 \mathrm{ppm}$. Detsri and Popanyasak [32] used a technique of layer-by-layer deposition to present the composite Ag NPS thin films as well as polyaniline. There was a change of color from orange-red to yellow when the films were exposed to ammonia solution.

Some of the chemicals in these works used to synthesize Ag NPs were, however, toxic to the environment, and excess chemicals were required to facilitate the reaction process. Natural products are of great interest particularly those derived from plants. Natural biopolymers, for instance, play a significant role in nanotechnology. This shows a great potential in various applications such as sensors, adsorption, catalysis, and antimicrobial activity [33-35], through green chemicals like polysaccharides, sugar, plant extract, and ascorbic acid [36] which acts as both a stabilizer and reducing agent. The disadvantage of using the chemicals, however, is that it requires a long reaction time for the completion of the reduction process for $20 \mathrm{~h}$ at room temperature [37]. This study reports green biosynthesis of Ag NPs through a durian fruit shell using a stabilizing and reducing reagent. The synthesized hybrid nanomaterial is effective in the sensing and monitoring of ammonia solution on the first encounter. Detection of ammonia that is dissolved is conducted on the basis of reagent color change in the presence of ammonia as seen in the absorption spectrometer. The material has the following specific characteristics: the response time is quick, the detection limits should be low, and it should be ecofriendly and easily available at a low cost, operates optimally at room temperature, requires no other reducing or stabilizing agents, functions optimally in an ambient environment and needs no air supply or oxygen, and requires no external stimuli such as illumination for reaction to initiate joule heating. UV-vis spectrophotometer was used in the monitoring of the kinetics of the synthesis of NPs while their morphology and size were confirmed through different techniques of analysis. The sensing characteristics of the NPs solutions were tested against the surging ammonia solution concentrations between 500 and $3000 \mathrm{ppm}$ through the monitoring of alterations in the amplitude and position of the LSPR with a UV-vis spectrophotometer.

\section{Experimental Procedure}

2.1. Chemicals. The chemicals for the experiment were sourced differently. Durian fruit was bought from a supermarket in Taif. Aqueous ammonia solution 25\% was procured from Merck, Germany, while the sodium hydroxide and silver nitrate were bought from Sigma Aldrich, USA. Besides these items, the other additional constituent was deionized water, whose purpose was to prepare the aqueous solution for the investigations. It is worth noting that the elements were of analytical grade and were used wholly without any further refinement or adulteration.

2.2. Preparing Durian Fruit Extracts. The process involved collecting and thoroughly rinsing fresh durian fruit in distilled water. $25 \mathrm{~g}$ was then picked and cut into tiny slices before being smashed and ground in a grinding mixer by adding some $100 \mathrm{ml}$ of Millipore water. Filtration of the raw extract was done using the Whatman filter paper (no. 1) before centrifugation for 10 minutes at $8000 \mathrm{rpm}$ to get rid of impurities, notably fiber. Observations made indicated that, following the centrifugation, a clear or transparent extract was obtained, and the measure of viscosity was $0.87 \mathrm{mPas}$. The aqueous solution was stored in a refrigerator to be used later.

2.3. Silver Nanoparticles Synthesis. For this experiment, $90 \mathrm{ml}$ of $10^{-3} \mathrm{M}$ of silver nitrate aqueous solution was mixed with $10 \mathrm{ml}$ of the extract. This was followed by adding sodium hydroxide droplets while stirring to regulate the $\mathrm{pH}$ to 10. The mixture was put in a dark room for several hours to settle. The solution was intermittently subjected to UV-vis spectroscopy measurements to gauge how the Ag NPs form. Ammonia sensing was then tested by further characterizing the synthesized silver nanoparticles. Those nanoparticles that remained were placed in amber bottles and stored at room temperature in a dark room.

2.4. Characterizing Synthesized Silver Nanoparticles. By using the UV-visible absorption spectrometer, specifically the Specord S100 from Analytica Jena $\mathrm{GmbH}$, the optical absorption spectra of these elements can be obtained. In this case, the spectra were collected at room temperature between 350 and $800 \mathrm{~nm}$ with an instrument resolution of $1 \mathrm{~nm}$. The study of the structure of these Ag nanoparticles is 
done using a scanning electron microscope model JEOL JSM-6390LV. Using this microscope, thin films of the sample were placed on grids that are copper carbon coated, and thereafter the recording of the images was done. The morphology of the particles was further investigated on a transmission electron microscope (TEM) by first collecting and washing them in ethanol and water before centrifugation at $15000 \mathrm{rpm}$. This was repeated a number of times to ensure that the protective shell of the fruit extract is removed to avail the biomolecules therein. Furthermore, the elemental silver that is existent in the sample was subjected to energy dispersive spectroscopy (EDS) together with TEM. An X-ray diffractometer, model RIGAKU MINIFLEX, with $\mathrm{Cu}-\mathrm{K} \alpha$ radiation line $\lambda=1.5405 \AA$ was used to determine the $\mathrm{XRD}$ patterns of the samples in a structural analysis. Complete scans of $2 \theta, 20^{\circ}-80^{\circ}$ with a step size of $0.05^{\circ}$ are conducted and the photoelectronic spectroscopy, model number XPS ESCALAB 210, was used to conduct the collection of silver from the surface of the nanoparticles.

2.5. The Test for Sensing Ammonia. The experiment was conducted by mixing $1 \mathrm{ml}$ of ammonia aqueous solution with $1 \mathrm{ml}$ of silver nanoparticles. Then, different concentrations of ammonia in a constant range between 0 and 3000 were mixed with an aqueous solution of $25 \%$ ammonia. Observations made included a change of color detected by naked eye and the detection of SPR nanoparticles when UVvis spectrophotometer was used. A spectral decline was observed on increase of ammonia concentrate at different levels. Further, there was a remarkable decrease in the UVvis spectrum when the quantity of ammonia was increased. The discussion section highlights these absorption intensity variations as well as their related processes at length.

\section{Results and Discussion}

3.1. Formation of Green Silver Nanoparticles. Simple, onestep, reproducible, cost-effective, high yield, and environment friendly NPs through green synthesis are becoming widespread and in high demand as a solution for a global problem, together with other environmental concerns [38-41]. This study carried out a green synthesis of Ag NPs using durian fruit shell extract. The most prevalent method for preparing stable Ag NPs is chemical reduction where colloidal dispersion occur in organic solvents or water [42]. Colloidal Ag with NPs particles of different diameters are yielded from the reduction of the $\mathrm{Ag}$ ions in aqueous solution [43]. The initial reduction of several complexes with silver ions leads to $\mathrm{Ag}$ atoms formation. The reaction is followed by the agglomeration of the metal ions into oligomeric clusters [44-47]. The solutions have an intense band of 380-400 $\mathrm{nm}$ and a yellow color when the colloidal particles are smaller than the visible light wavelength. The intense band could be lesser than the smaller bands with longer wavelengths in the absorption spectrum [48]. The Ag NPs synthesis involves three key steps that are subject to evaluation on the basis of the perspectives of green chemistry. The steps include solvent media selection, selection of reducing agents that are environmentally benign, and selection of substances that are not toxic to stabilize the silver NPs. The green chemistry method of synthesizing Ag NPs through chemical reduction, therefore, has been widely used in this study.

On adding $\mathrm{NaOH}(1 \mathrm{M})$ drop-wise to the mixture of durian organic product shell containing $\mathrm{Ag}^{+}$particles $\left(\mathrm{AgNO}_{3}, 1 \mathrm{mM}\right)$, the color of the response blend became yellowish and afterwards dim dark colored. The formation of silver nanoparticles was proved by the adjustment in color of the arrangement after expansion of $\mathrm{AgNO}_{3}$ from colorless to light yellowish shading, at that point to the dim darker shading. Subsequent to adding silver nitrate arrangement to concentrate of durian organic product shell, the shades of the blends changed from colorless to orange-yellow as shown in Figure 1(a) demonstrating the development of silver nanoparticles. The colour of the solutions got darker with increase in time, which means the higher amount of silver nanoparticles were obtained. The various shades of silver nanoparticles are identified with the excitation impact of surface plasmon resonance [49-51].

Development of silver nanoparticles by decreasing $\mathrm{Ag}^{+}$particles was deliberately checked by UV-vis spectroscopy at 30,60,90,120,150,180 and $210 \mathrm{~min}$. By recording the changes in absorbance as a function of time, the kinetic of development of silver nanoparticles formation was checked. [52,53], and the accumulation of the absorbance spectra is shown in Figure 1(b). This information uncovers a few significant discoveries which can be exhibited as follows: there was an increase in the height of the peak with increase of time. (i) At the beginning of the period of response length (after $90 \mathrm{~min}$ ), the plasmon band is expanded, and straightforward test for silver particle shows low transformation of silver particles to metallic silver nanoparticles at this span, (ii) drawing out the response term up to $180 \mathrm{~min}$ prompts remarkable upgrade in the plasmon force demonstrating that a lot of silver particles are diminished and utilized for bunch development, and (iii) further increment in the response term up to $210 \mathrm{~min}$ results in negligible decrement in the retention power which could be credited to some conglomeration of the shaped silver nanoparticles. The last absorbance pinnacle of the nanoparticles arrangement stands out unequivocally from the normal absorbance most extreme at $394 \mathrm{~nm}$, which is usually exhibited as the quality of fruitful silver nanoparticles amalgamation. The absorbance peak normally emerges because of the excitation of confined surface plasmon motions of the conduction electrons if there should arise an occurrence of valuable metal nanoparticles, for example, gold and silver.

The arrangement of $\mathrm{Ag} \mathrm{NPs}$ from $\mathrm{Ag}^{+}$particles isstarted by the compound responses within polysaccharide (polyols) with the general formula $\mathrm{C}_{x}\left(\mathrm{H}_{2} \mathrm{O}\right)_{y}$ and ascorbic corrosive $\left(\mathrm{C}_{6} \mathrm{H}_{8} \mathrm{O}_{6}\right)$ as decreasing agents, both present in durian organic product shell extract [54]. The general response can be composed as follows: 

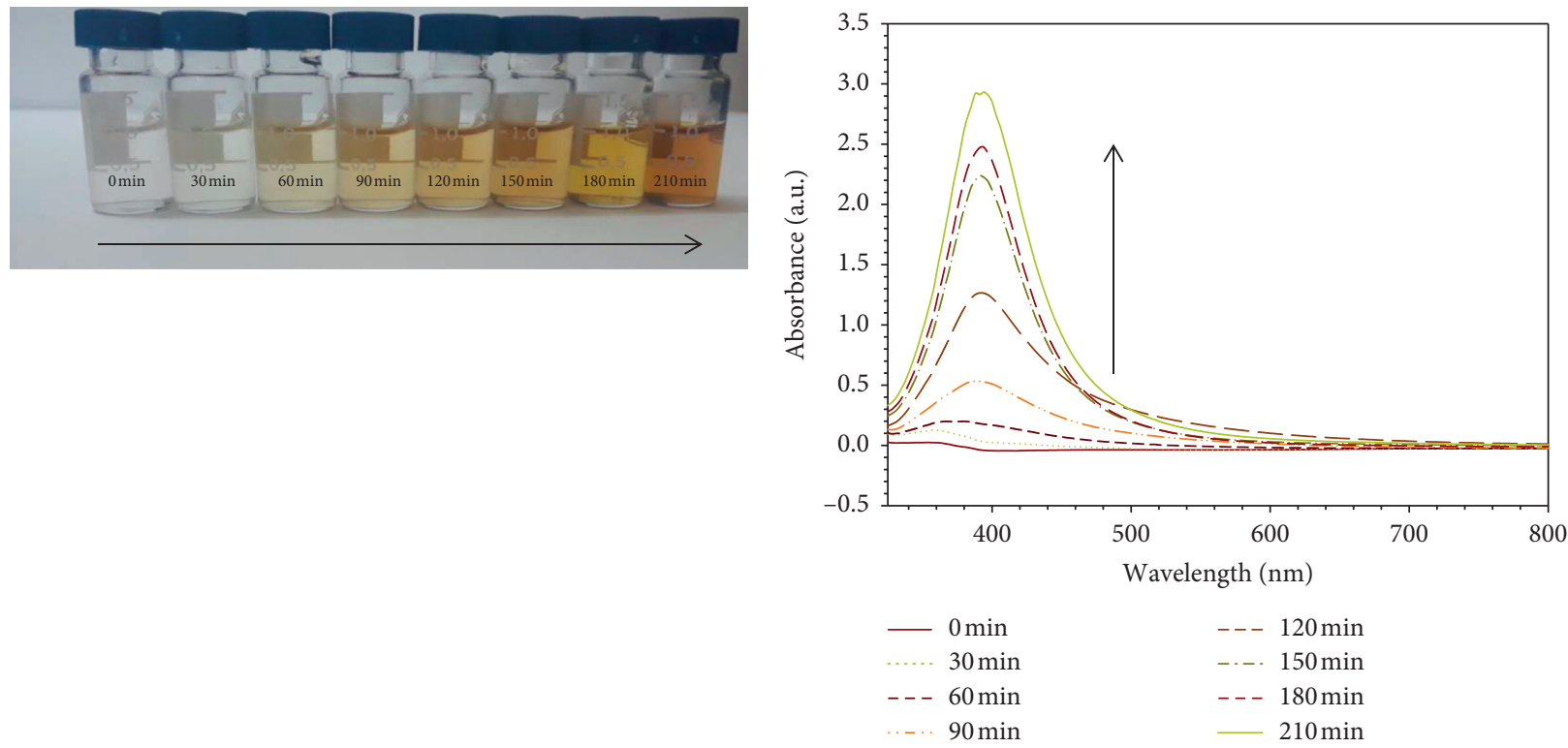

(a)

(b)

Figure 1: The appearances of silver nitrate solution and AgNPs colloids synthesized (a), and the UV-vis spectra of the formed AgNPs at various times of $0,30,60,90,120,150,180$ and $210 \mathrm{~min}(\mathrm{~b})$.

$$
\begin{aligned}
2 \mathrm{Ag}^{+}+ & \mathrm{C}_{x}\left(\mathrm{H}_{2} \mathrm{O}\right)_{y}+\mathrm{H}_{2} \mathrm{O} \longrightarrow 2 \mathrm{Ag}^{\circ}+\left(\mathrm{C}_{x} \mathrm{H}_{2 y-1} \mathrm{O}_{y+1}\right)^{-} \\
& +3 \mathrm{H}^{+} \\
2 \mathrm{Ag}^{+}+ & \mathrm{C}_{6} \mathrm{H}_{8} \mathrm{O}_{6} \longrightarrow 2 \mathrm{Ag}^{\circ} \\
& \left.+\mathrm{C}_{6} \mathrm{H}_{8} \mathrm{O}_{6} \text { (diydroxy ascorbic acid }\right)+2 \mathrm{H}^{+}
\end{aligned}
$$

It is clear that every increase in the concentration of $\mathrm{OH}^{-}$ ions forwarded the reaction and resulted in an increase in the production of silver nanoparticles. This was confirmed by a higher $\mathrm{pH} 10$ yield.

\subsection{Characteristics of the Green Synthesized Silver} Nanoparticles. The mixture of the reaction was centrifuged with $4000 \mathrm{rpm}$ for $20 \mathrm{~min}$ and the precipitant was centrifuged after being washed with running water. At that point, the pellets were gathered and dried in hot air oven at a legitimate temperature of $35-40^{\circ} \mathrm{C}$. Dried nanoparticles were gathered and utilized for additional portrayal and application studies. Nanoparticles are, for the most part, portrayed by contributing their size, shape, and surface territory. Homogeneity in these properties brings about the progression in utilization of nanoparticles. The portrayal of silver nanoparticle is performed by utilizing different instrumental procedures.

Morphological character and size subtleties of the incorporated silver nanoparticle were given by SEM pictures, Figure 2(a). This size variety in the nanoparticles is because of the nearness of proteins or different atoms from extract, which were bound at the outside of the nanoparticles. Furthermore, the outcome demonstrated that the blended silver nanoparticles were round fit as a fiddle. We have completed transmission electron minute (TEM) concentrate to get data about the inward structure (size and morphology) of the biosynthesized Ag nanoparticles. Our TEM picture in Figure 2(b) likewise shows that the the silver nanoparticles fabricated after 210 min utilizing fruit extracts are covered by a thin layer of capping materials. TEM picture shows circular particles of small size. Figure 2(c) shows the molecule size circulation histogram of the size conveyance of biosynthesized Ag nanoparticles estimated from TEM study. The TEM figure outlines that those nanoparticles measurement fluctuate between 20 and $30 \mathrm{~nm}$. The normal distance across silver nanoparticles was seen as around $25 \mathrm{~nm}$ with practically circular fit as a fiddle shape and monodispersed in nature. Comparative SAED design was acquired with silver nanoparticles blended utilizing aloe vera [55].

EDS range recorded from the silver nanoparticles is shown in Figure 2(d), separately. EDS profile shows solid silver sign. This pinnacle was framed because of the impact of surface plasmon reverberation of green-blended nanoparticle [56]. It was shown that arrangement of silver nanoparticles utilizing natural product shell separates. Other minor pinnacles, for example, $\mathrm{C}$ and $\mathrm{O}$, were seen at different retention vitality levels. These may happen from organic product shell extract because of the nearness of moieties of phytochemicals constituents. These parts engaged with the balancing out and topping agent on the outside of nanoparticles. Comparable outcomes were accounted for on green blend of silver nanoparticles [57]. Together with TEM pictures, Shankar et al. [58] detailed that nanoparticles combined utilizing plant extracts are encompassed by a slight layer of some topping natural material from organic product.

The spectrum of XPS displays a characteristic Ag peak on the NPs surface which indicates that Ag NPs are synthesized successfully through the durian fruit extraction as shown in 


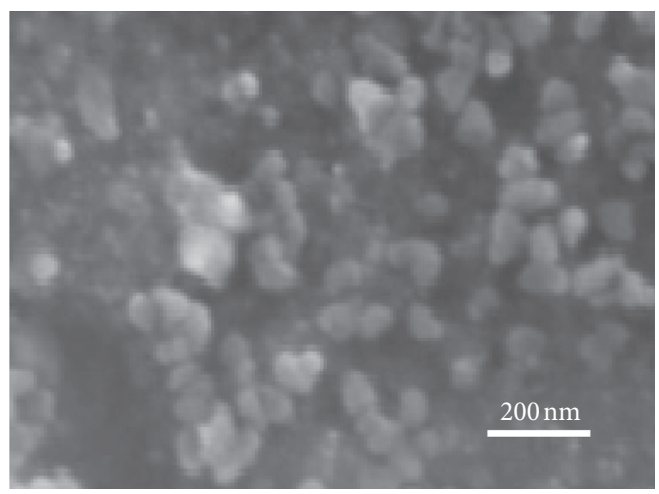

(a)

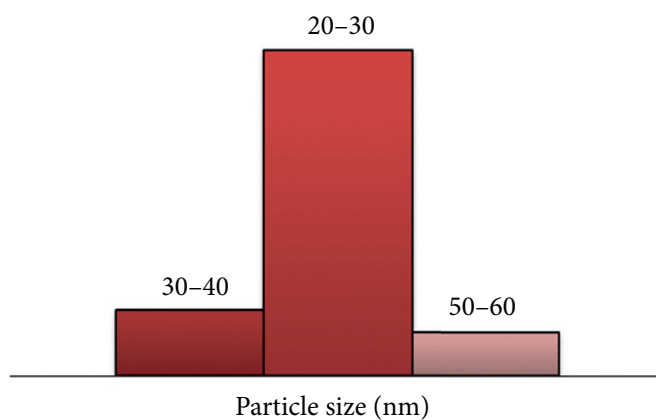

(c)

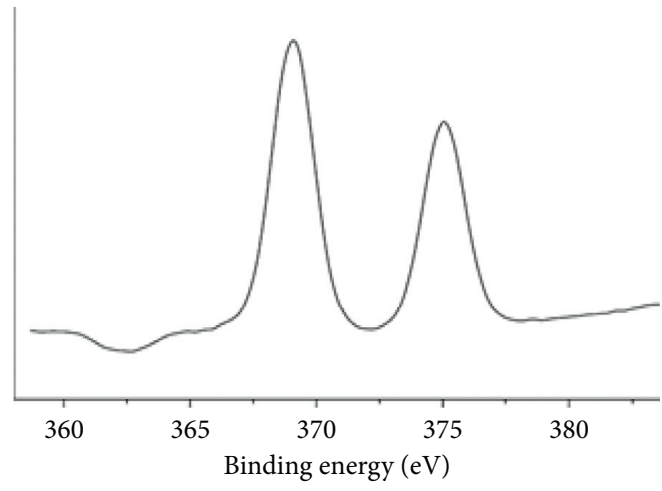

(e)

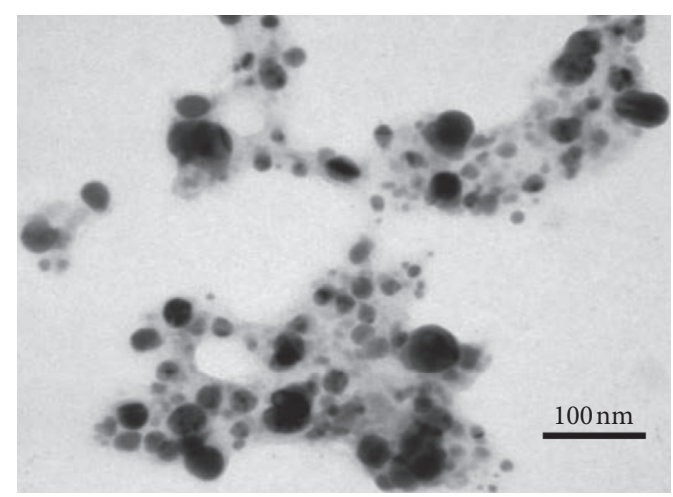

(b)

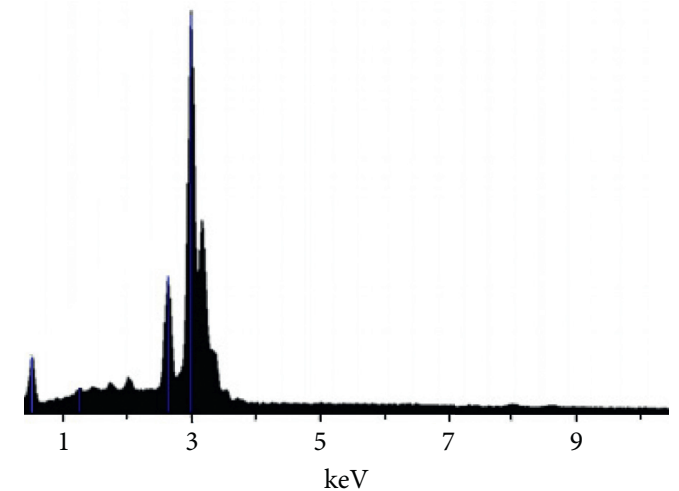

(d)

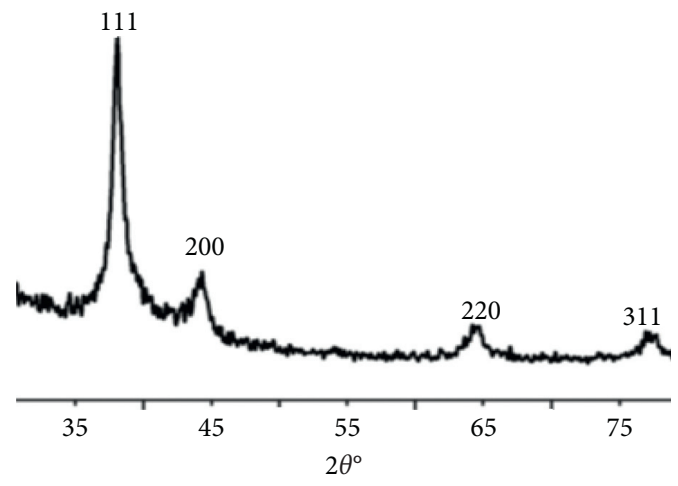

(f)

FIgURE 2: Characterization of silver nanoparticles formed with $90 \mathrm{~mL}$ of $10-3 \mathrm{M}$ aqueous $\mathrm{AgNO}_{3}$ solution and durian fruit shell, (a) SEM micrograph, (b) TEM micrograph, (c) diameter particles diameter with counts, and (d) EDS spectrum, (e) XPS spectrum, and (f) XRD analysis.

Figure 2(e). When the reaction is starting, the $\mathrm{Ag}^{+}$are reduced precipitating the formation of Ag atoms. The nuclei of the Ag atoms rapidly form small NPs due to the reduced capping agent that is physically adsorbed on the NPs surface. The small NPs that are formed aggregate to form larger NPs due to a higher surface energy and high density of the smaller NPs at the start of the chemical process. The large particles that are formed, however, are unstable and can be degraded into smaller components. As the reaction proceeds, more $\mathrm{Ag}^{+}$are reduced to form $\mathrm{Ag}$ atoms, thus causing a decrease at the start and a later increase at particular reaction time and then finally stabilizing [59].
Figure 2(f) shows the patterns of X-ray diffraction of the Ag NPs. The peaks are seen at various values which include $2 \theta$ values $37.8^{\circ}, 43.96^{\circ}, 64.34^{\circ}$, and $77.48^{\circ}$ that pertain $\mathrm{Ag}$ NPs. The planes (111), (200), (220), and (311) from the indexed peaks correspond to the silver face-centered cubic structure (JCPDS no.893722). The Ag NPs gram size was estimated from the XRD width peak at $2 \theta=37.8^{\circ}$. Scherer's formula was used to estimate the average NP size which was found to be $21 \mathrm{~nm}$. Average grain size of the nanoparticle was found to be $21 \mathrm{~nm}$ using Scherer's formula, $d_{\text {avg }}=((0.9 \lambda) / \beta \cos \theta)$, where $\lambda$ is the wavelength of $\mathrm{CuK}_{\alpha 1}$ radiation, $d_{\mathrm{avg}} i$ is the average size of the grain, $\beta$ is the entire 
width at half maximum (FWHM), and $\theta$ represents the angle of diffraction [60-62].

3.3. Ammonia Sensor Properties. Innovative technologies have been adopted in conducting the research for ammonia detection using ammonia sensors because of its functional applications in the industrial sector and its toxic nature to both humans and other living beings. The wide applications of ammonia in industrial applications create a lot of demand for the development of ammonia sensors as they are used for the purposes of its monitoring in a diverse range of applications [63-65]. In order to obtain the UV-vis spectra for each level, the color combinations were observed as indicated in Figure 3(a), with the color of the solution changing from brown to clear. With an increase in the concentration of ammonia, the color of the Ag NPs changes and the decreases in the intensity of absorption are detectable by the naked eye.

Optical measurement is the most ideal way of conducting a sensor study of an ammonia solution. Ag NP absorbance spectra studies contain different levels of ammonia concentrations and are documented as a function of varying concentrations of ammonia ranges between 0 and $3000 \mathrm{ppm}$ as indicated in Figure 3(b), while the values of ammonia concentration absorption is indicated in Figure 3(c). Different silver nanoparticles colloidal solutions are required for each measurement. Remarkably, the LSPR highest concentration for Ag NPs moves from $391 \mathrm{~nm}$ to $403 \mathrm{~nm}$ when ammonia solution is added to it. The difference in the Ag NPs SPR band is caused by the change in the surrounding medium's dialectic constant and the distance of interparticles [66].

Efforts for enhancement include data processing and considering the absorbance ratio of ammonia concentration (Abs391/403) as indicated in Figure 4. According to the ratio of absorbance, the concentration of ammonia linearity ranges from 500 to $3000 \mathrm{ppm}$. In such cases, the ration of absorbance for (Abs391/Abs403) is investigated and indicated as an element of ammonia concentration illustrating a relationship that is linear with a correlation factor $R^{2}$ which is an equivalent of 0.9831 , a figure which compares favorably to previous reports [7]. Absorbance ratios outputs a concentration of ammonia linearity within the range of 500 to $3000 \mathrm{ppm}$, and this, therefore, is an illustration that an analysis of the absorption spectra of $391 \mathrm{~nm}$ is possible and the set Ag NPs are used as an optical sensor. Therefore, these results enable us to make a conclusion that Ag NPs have a high capability to detect lower ammonia concentrations within solutions (of up to $3000 \mathrm{ppm}$ from our study) and thus are ideal optical sensors that we can deploy in ammonia sensing.

In the determination of the time needed to complete a complexing reaction, commonly referred to as the retention time, the silver nanoparticles colloidal solution is measured in a test tube where various ammonia levels are then added, in the range of 1000, 2000, and $3000 \mathrm{ppm}$. Observing the resultant output, the maximum absorbance recorded using the UV-vis spectrophotometer is shown in Figure 5. In
Figure 6, one requires a minimum of $25 \mathrm{~nm}$ to gain approximately $95 \%$ completion of the complex reaction, notwithstanding the amount of ammonia used.

3.4. Reaction of NPs with Ammonia. When $\mathrm{Ag}^{+}$ions are mixed with ammonia, a coordination complex $\mathrm{Ag}\left(\mathrm{NH}_{3}\right)_{2}{ }^{+}$is formed. When silver colloids come into contact with an ammonia solution, $\mathrm{Ag}\left(\mathrm{NH}_{3}\right)_{2}{ }^{+}$, they form a coordination complex between them, increasing the Ag NPs surface charge, which in turn creates a repulsion force between the particles thereby increasing the water content in the surrounding. The outcome isolates hydrophilic particles with a blue shift spectrum, as shown in Figure 7. Nanoparticles decolorization occurs as a result of the manipulation of the $\mathrm{Ag}$ NPs SPR through the formation of the $\mathrm{Ag}\left(\mathrm{NH}_{3}\right)_{2}{ }^{+}$. Complex $\mathrm{Ag}\left(\mathrm{NH}_{3}\right)_{2}{ }^{+}$forms Ag NPs to break away through their transformation into smaller nanoparticles with blue shift. The formed complex diminishes the quantity of $\mathrm{Ag}$ NPs thereby successfully decreasing its color intensity. Consequently, the plasmonic bands of silver nanoparticles are directly affected by the amount of $\mathrm{Ag}\left(\mathrm{NH}_{3}\right)_{2}{ }^{+}$. The implication of this is that the more the ammonia, the higher the amounts of $\mathrm{Ag}\left(\mathrm{NH}_{3}\right)_{2}{ }^{+}$complex and hence the lower the Ag NPs amount. Therefore, a reduction in a silver nanoparticles plasmonic band is unavoidable $[67,68]$.

3.5. Applications of Spectrophotometric Method. The developed sensors need to be tested for their applicability in determining ammonia levels in actual samples and therefore the most efficient way to do this is by using the spectrophotometric approaches. To achieve this, two samples of marine water and bottled water are spiked with varying concentrations of ammonia and an analysis is done. The results that are used for determining ammonia and recovery for the spike samples are as indicated in Table 1. For each of the three concentrations, three measurements are conducted, giving a good outcome between the value estimations in the analysis in the spectrophotometric approaches. The recovery is recorded at the range of $89.66-101.44 \%$. Outcomes of metallic nanoparticles unique LSPR properties indicate that they are simple and of low cost, have a quick response time and great reproducibility, and are highly sensitive, characteristics which make them very useful.

3.6. Stability of the Fabricated AgNPs. Figure 8 shows a study that was conducted for four months. Its outcome indicates that stability is constant for the whole duration of four months. Likewise, the fabricated silver nanoparticles absorbance decreases to 2.582 a.u., while the wavelength slightly moves effectively from $391 \mathrm{~nm}$ to $394 \mathrm{~nm}$. When preparing the silver nanoparticles mixture, large-sized agglomerates are formed through the Vander Waals's principle or the Colomb's force from the individual particles. A reducing agent is added to the mixture to inhibit the agglomeration of the small particles. Usually the durian fruit shell extract is used as the stabilizing reducing reagent as it creates a shielding layer on the surface of the particle, ensued 


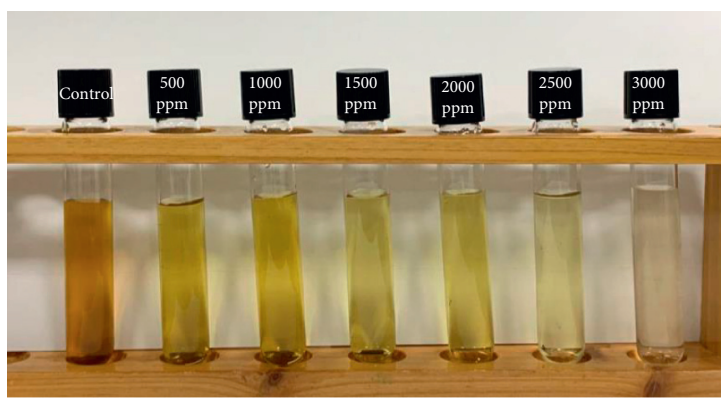

(a)
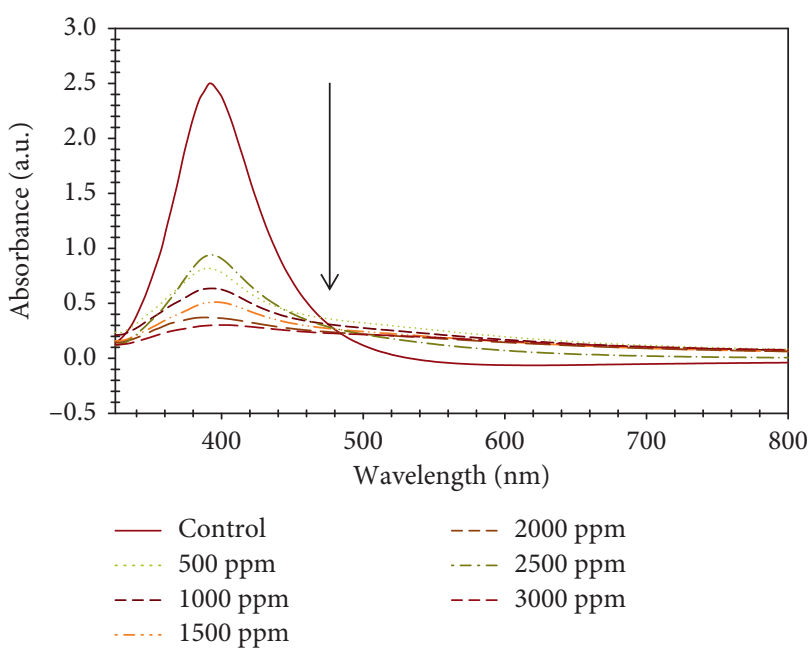

(b)

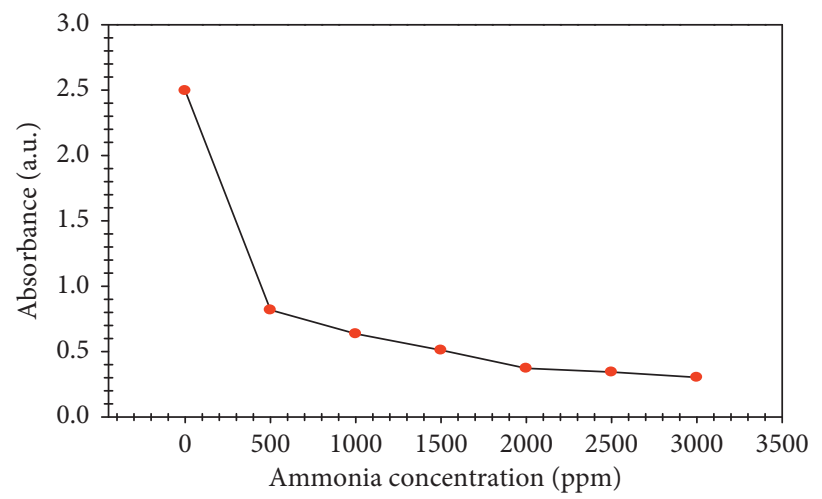

(c)

FIgURE 3: The effect of ammonia on the green fabricated silver nanoparticles; different levels of ammonia were added to the colloidal solution of Ag NPs containing a constant level of the nanoparticles (a). Change in the UV-visible spectra of silver nanoparticles as a function of different concentrations of ammonia from 0 to $3000 \mathrm{ppm}$ (b). Plot of absorbance versus ammonia concentration (c).

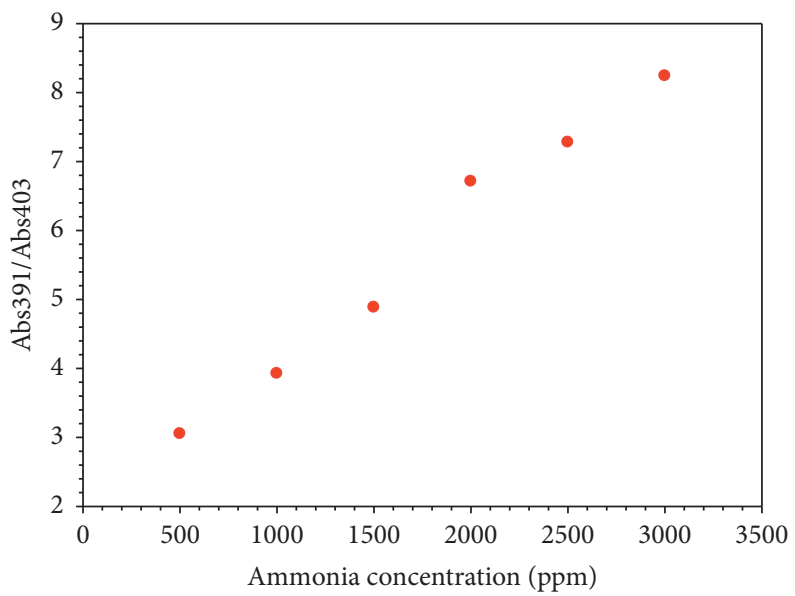

Figure 4: Absorbance ratio at $391 \mathrm{~nm}$ and $403 \mathrm{~nm}$, which displays a linear relationship as a function of different ammonia concentrations (500 ppm to $3000 \mathrm{ppm}$ ) with a correlation factor $R_{2}$ equal to 0.9831 . 


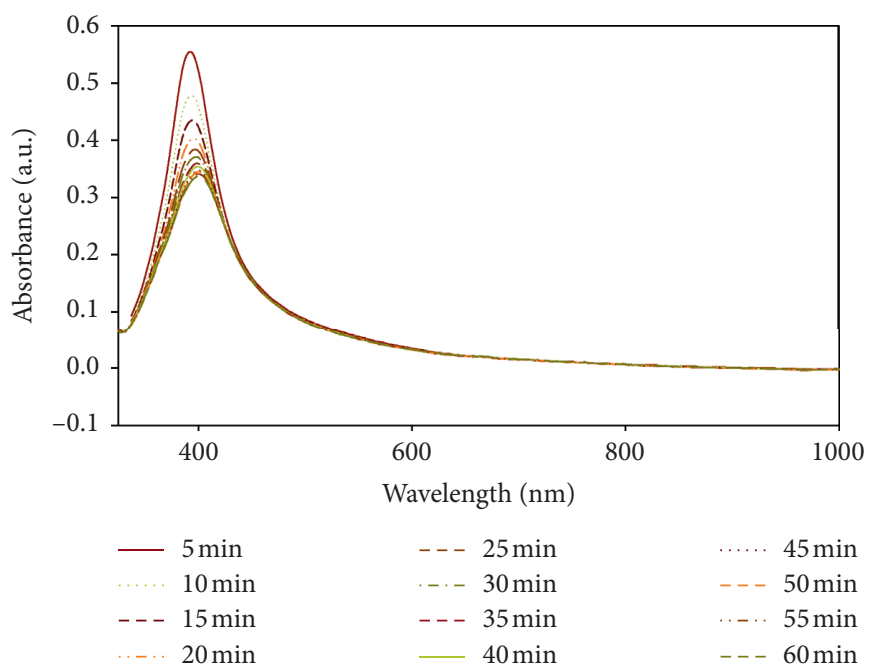

(a)

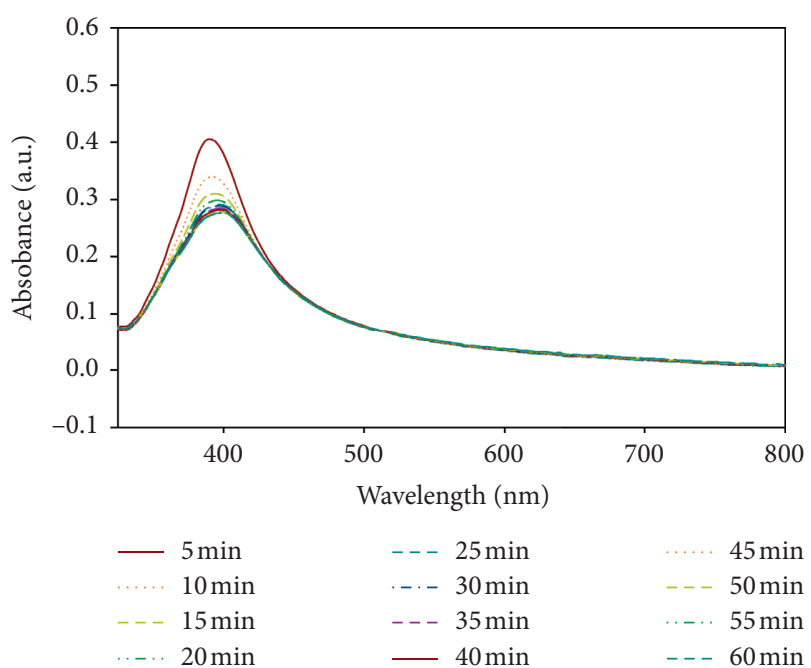

(b)

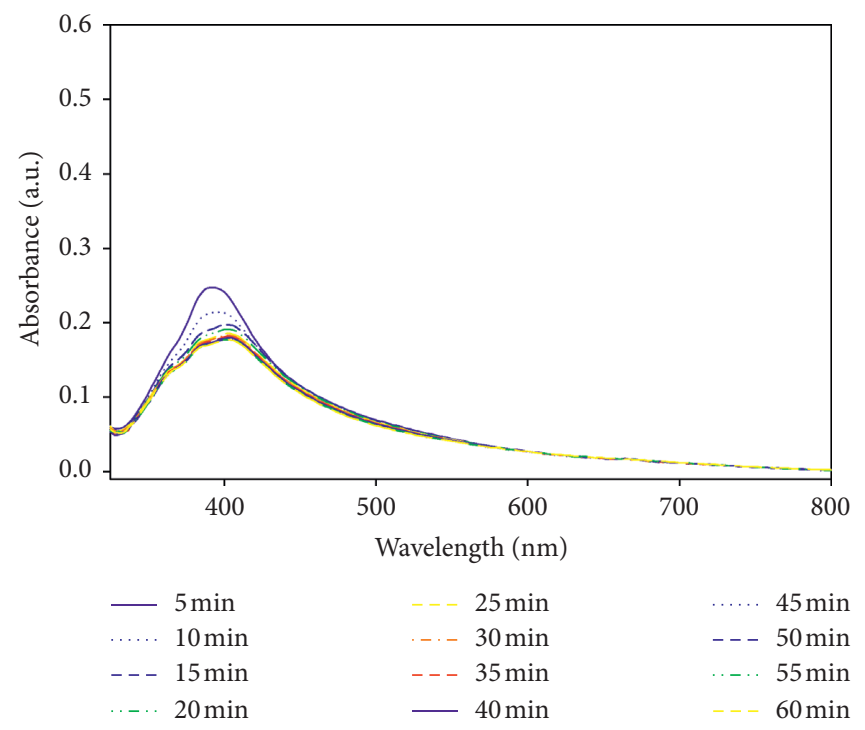

(c)

FIGURE 5: Effect of time (5-60 min) on the absorbance of the green fabricated silver nanoparticles after adding different amounts of ammonia solution: (a) $1000 \mathrm{ppm}$, (b) $2000 \mathrm{ppm}$, and (c) $3000 \mathrm{ppm}$.

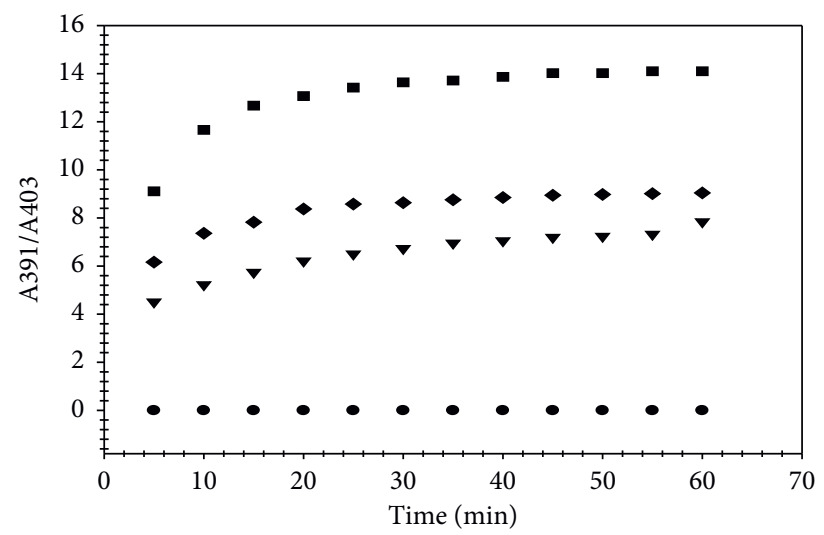

FIGURE 6: Absorbance ratio of the fabricated silver nanoparticles versus elapsed time in the presence of different concentrations of ammonia: 0 ppm $(\bullet), 1000$ ppm $(\boldsymbol{\nabla}), 2000$ ppm $(\bullet), 3000$ ppm (汭. 


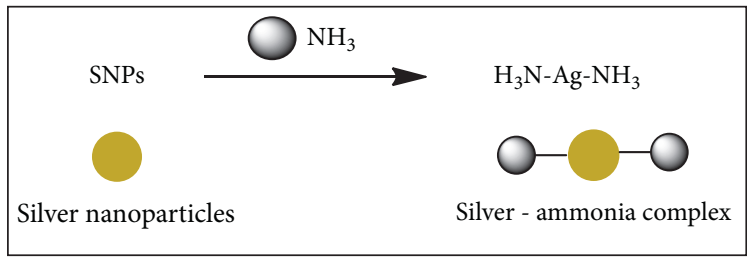

FIGURE 7: Reaction mechanism of silver nanoparticles with ammonia.

TABLE 1: Determination of ammonia in real sample using the synthesized silver nanoparticles.

\begin{tabular}{lccc}
\hline Type of sample & Ammonia added $(\mathrm{ppm})$ & Ammonia found $(\mathrm{ppm})$ & Recovery $(\%)$ \\
\hline \multirow{2}{*}{ Bottle water } & 900 & $913 \pm 0.6$ & 101.44 \\
& 1800 & $1811 \pm 1.9$ & 100.61 \\
\multirow{2}{*}{ Marine water } & 900 & $807 \pm 0.8$ & 89.66 \\
& 1800 & $1689 \pm 2.1$ & 93.83 \\
\hline
\end{tabular}

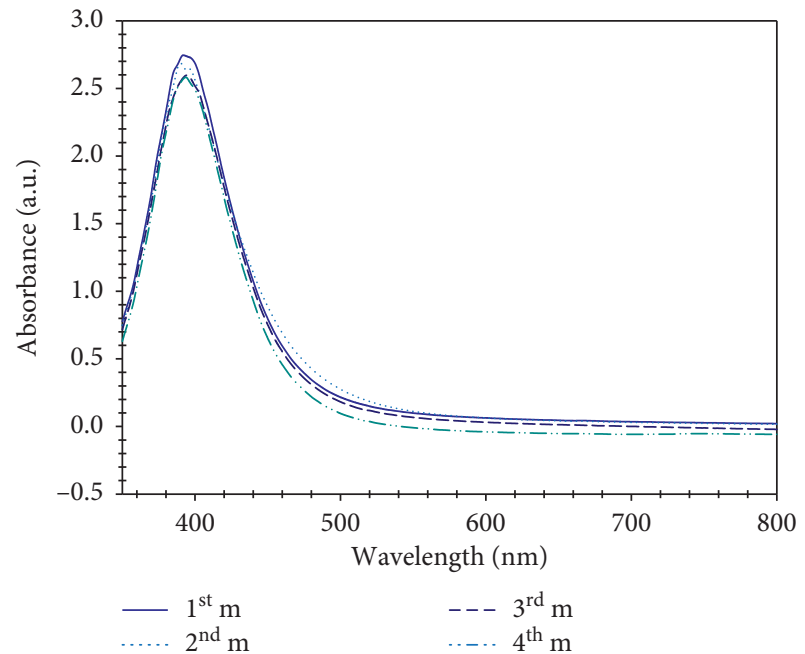

FIGURE 8: UV-vis spectra of the fabricated silver nanoparticles to check the stability of the fabricated silver nanoparticles for four months.

by a strong absorption of the reducing agent on the surface of the silver nanoparticles, thus ensuring that better stabilization is achieved.

\section{Conclusions}

The current study uses the durian fruit shell extract to synthesize silver nanoparticles from silver nitrate, a technique that is commonly adaptable in principles of green chemistry. The durian fruit extract performs the functions of stabilizing and reducing the synthesized silver nanoparticles. The durian fruit shell agent used influences the synthesis of the nanoparticles to increase with the silver nitrate concentration and the reaction time. Since there is a possibility of controlling the size of the nanoparticles, this method is ideal for large scale industrial applications of monodispersed and circular nanoparticles of approximately $25 \mathrm{~nm}$ given the fact that low-cost biopolymer is available. The decline in the plasmonic features of the Ag NPs can be attributed to the presence of the oxidizing agent in the ammonia solution which has the ability to coagulate into $\mathrm{Ag}$ $\left(\mathrm{NH}_{3}\right)_{2}{ }^{+}$with $\mathrm{Ag}$ ions. The characteristics of the Ag NPs make it possible for the ammonia colorimetric detection and could therefore be used for optical sensing in healthcare diagnostics to detect low levels of ammonia in the human body. These attributes include the localized SPR property, simplicity, sensitivity, affordability, and its quick turnaround time.

\section{Data Availability}

The data used in this paper are available upon reasonable request from the corresponding author.

\section{Additional Points}

No animals/humans were used for studies that are the basis of this research.

\section{Conflicts of Interest}

The author declares that there are no conflicts of interest regarding the publication of this paper.

\section{Acknowledgments}

The author acknowledges the support of Taif University Researchers Supporting Project number TURSP-2020/136, Taif University, Taif, Saudi Arabia.

\section{References}

[1] P. Warneck, Chemistry of the Natural Atmosphere, Elsevier, Amsterdam, Netherlands, 1999.

[2] E. Abatenh, B. Gizaw, Z. Tsegaye, and T. Genene, "Microbial function on climate change-a review," Open Journal of Environmental Biology, vol. 3, no. 1, pp. 1-7, 2018.

[3] C. E. Boyd, Nitrogen, in Water Quality, pp. 269-290, Springer, Berlin, Germany, 2020.

[4] K. Rousk, M. Vestergård, and S. Christensen, "Are nitrous oxide emissions and nitrogen fixation linked in temperate bogs?” Soil Biology and Biochemistry, vol. 123, pp. 74-79, 2018.

[5] T. Shah, S. Lateef, and M. A. Noor, "Carbon and nitrogen cycling in agroecosystems: an overview," in Carbon and 
Nitrogen Cycling in Soil, pp. 1-15, Springer, Berlin, Germany, 2020.

[6] X. Yang, F. Ling, J. Su et al., "Insights into the role of cation vacancy for significantly enhanced electrochemical nitrogen reduction," Applied Catalysis B: Environmental, vol. 264, Article ID 118477, 2020.

[7] L. Chetia, D. Kalita, and G. A. Ahmed, "Synthesis of Ag nanoparticles using diatom cells for ammonia sensing," Sensing and Bio-Sensing Research, vol. 16, pp. 55-61, 2017.

[8] Y. Jegal and Y. Kim, "Industrial chemicals and acute lung injury with a focus on exposure scenarios," Current Respiratory Medicine Reviews, vol. 12, no. 1, pp. 44-55, 2016.

[9] J. G. Speight, Handbook of Petrochemical Processes, CRC Press, Boca Raton, FL, USA, 2019.

[10] C. Pijolat, C. Pupier, M. Sauvan, G. Tournier, and R. Lalauze, "Gas detection for automotive pollution control," Sensors and Actuators B: Chemical, vol. 59, no. 2-3, pp. 195-202, 1999.

[11] Y. Chang, Z. Zou, C. Deng et al., "The importance of vehicle emissions as a source of atmospheric ammonia in the megacity of Shanghai," Atmospheric Chemistry and Physics, vol. 16, no. 5, pp. 3577-3594, 2016.

[12] W. Ament, J. R. Huizenga, E. Kort, T. W. Van Der Mark, R. G. Grevink, and G. J. Verkerke, "Respiratory ammonia output and blood ammonia concentration during incremental exercise," International Journal of Sports Medicine, vol. 20, no. 2, pp. 71-77, 1999.

[13] L. Narasimhan, W. Goodman, and C. K. N. Patel, "Correlation of breath ammonia with blood urea nitrogen and creatinine during hemodialysis," Proceedings of the National Academy of Sciences, vol. 98, no. 8, pp. 4617-4621, 2001.

[14] R. Alam, "Study on removal of ammonia from the waste water of Fenchuganj natural gas fertilizer factory," Dissertation Thesis, Bangladesh University of Engineering and Technology, Dhaka, Bangladesh, 2006.

[15] K. Zakrzewska, "Mixed oxides as gas sensors," Thin Solid Films, vol. 391, no. 2, pp. 229-238, 2001.

[16] M. Sahm, A. Oprea, N. Bârsan, and U. Weimar, "Water and ammonia influence on the conduction mechanisms in polyacrylic acid films," Sensors and Actuators B: Chemical, vol. 127, no. 1, pp. 204-209, 2007.

[17] Y. Shen and P. Prasad, "Nanophotonics: a new multidisciplinary frontier," Applied Physics B, vol. 74, no. 7-8, pp. 641-645, 2002.

[18] C. Molins-Legua, S. Meseguer-Lloret, Y. Moliner-Martinez, and P. Campíns-Falcó, "A guide for selecting the most appropriate method for ammonium determination in water analysis," TrAC Trends in Analytical Chemistry, vol. 25, no. 3, pp. 282-290, 2006.

[19] J. Li and P. K. Dasgupta, "Chemiluminescence detection with a liquid core waveguide: determination of ammonium with electrogenerated hypochlorite based on the luminol-hypochlorite reaction," Analytica Chimica Acta, vol. 398, no. 1, pp. 33-39, 1999.

[20] B. Wiley, Y. Sun, B. Mayers, and Y. Xia, "Shape-controlled synthesis of metal nanostructures: the case of silver," Chemistry-A European Journal, vol. 11, no. 2, pp. 454-463, 2005.

[21] M.-R. Xie, Y. Cai, Y.-Q. Liu, and Z.-Y. Wu, "Sensitive colorimetric detection of $\mathrm{Pb}^{2+}$ by geometric field amplification and surface plasmon resonance visualization," Talanta, vol. 212, Article ID 120749, 2020.

[22] I. C. Sulaiman, B. W. Chieng, M. J. Osman et al., "A review on colorimetric methods for determination of organophosphate pesticides using gold and silver nanoparticles," Microchimica Acta, vol. 187, no. 2, pp. 1-22, 2020.
[23] S. Prajapati, "Nanotechnology-based sensors," in BiopolymerBased Formulations, pp. 237-262, Elsevier, Amsterdam, Netherlands, 2020.

[24] K. A. Willets and R. P. Van Duyne, "Localized surface plasmon resonance spectroscopy and sensing," Annual Review of Physical Chemistry, vol. 58, pp. 267-297, 2007.

[25] P. Slepička, N. Slepickova Kasalkova, J. Siegel, Z. Kolska, and V. Svorcik, "Methods of gold and silver nanoparticles preparation," Materials, vol. 13, no. 1, pp. 1-22, 2020.

[26] T. Ritthichai and V. Pimpan, "Ammonia sensing of silver nanoparticles synthesized using tannic acid combined with UV radiation: effect of UV exposure time," Journal of King Saud University-Science, vol. 31, no. 2, pp. 277-284, 2019.

[27] S. Zeng, D. Baillargeat, H.-P. Ho, and K.-T. Yong, "Nanomaterials enhanced surface plasmon resonance for biological and chemical sensing applications," Chemical Society Reviews, vol. 43, no. 10, pp. 3426-3452, 2014.

[28] N. Yang, X.-F. Wei, and W.-H. Li, "Sunlight irradiation induced green synthesis of silver nanoparticles using peach gum polysaccharide and colorimetric sensing of $\mathrm{H}_{2} \mathrm{O}_{2}$," Materials Letters, vol. 154, pp. 21-24, 2015.

[29] P. Manivel and M. Ilanchelian, "Selective and sensitive colorimetric detection of $\mathrm{Hg}^{2+}$ at wide $\mathrm{pH}$ range using green synthesized silver nanoparticles as probe," Journal of Cluster Science, vol. 28, no. 3, pp. 1145-1162, 2017.

[30] J. Song, O. Ma, S. Zhang, Y. Guo, and C. Dong, "Preparation of silver nanoparticles reduced by formamidinesulfinic acid and its application in colorimetric sensor," Journal of Cluster Science, vol. 27, no. 4, pp. 1203-1212, 2016.

[31] S. T. Dubas and V. Pimpan, "Green synthesis of silver nanoparticles for ammonia sensing," Talanta, vol. 76, no. 1, pp. 29-33, 2008.

[32] E. Detsri and J. Popanyasak, "Fabrication of silver nanoparticles/polyaniline composite thin films using layer-by-layer self-assembly technique for ammonia sensing," Colloids and Surfaces A: Physicochemical and Engineering Aspects, vol. 467, pp. 57-65, 2015.

[33] S. Pandey, "A comprehensive review on recent developments in bentonite-based materials used as adsorbents for wastewater treatment," Journal of Molecular Liquids, vol. 241, pp. 1091-1113, 2017.

[34] S. Pandey, "Highly sensitive and selective chemiresistor gas/ vapor sensors based on polyaniline nanocomposite: a comprehensive review," Journal of Science: Advanced Materials and Devices, vol. 1, no. 4, pp. 431-453, 2016.

[35] S. Pandey and K. K. Nanda, "Au nanocomposite based chemiresistive ammonia sensor for health monitoring," Acs Sensors, vol. 1, no. 1, pp. 55-62, 2016.

[36] S. Ganaie, T. Abbasi, J. Anuradha, and S. A. Abbasi, "Biomimetic synthesis of silver nanoparticles using the amphibious weed ipomoea and their application in pollution control," Journal of King Saud University-Science, vol. 26, no. 3, pp. 222-229, 2014.

[37] P. Raveendran, J. Fu, and S. L. Wallen, "Completely "green" synthesis and stabilization of metal nanoparticles," Journal of the American Chemical Society, vol. 125, no. 46, pp. 13940-13941, 2003.

[38] P. Tippayawat, N. Phromviyo, P. Boueroy, and A. Chompoosor, "Green synthesis of silver nanoparticles in aloe vera plant extract prepared by a hydrothermal method and their synergistic antibacterial activity," PeerJ, vol. 4, Article ID e2589, 2016.

[39] A. Allafchian, S. Z. Mirahmadi-Zare, S. A. H. Jalali, and M. R. Vahabi, "Green synthesis of silver nanoparticles using 
phlomis leaf extract and investigation of their antibacterial activity," Journal of Nanostructure in Chemistry, vol. 6, no. 2, pp. 129-135, 2016.

[40] M. E. Palanco, K. Bo Mogensen, M. Gühlke, Z. Heiner, J. Kneipp, and K. Kneipp, "Templated green synthesis of plasmonic silver nanoparticles in onion epidermal cells suitable for surface-enhanced Raman and hyper-Raman scattering," Beilstein Journal of Nanotechnology, vol. 7, no. 1, pp. 834-840, 2016.

[41] S. Ahmed, Saifullah, M. Ahmad, B. L. Swami, and S. Ikram, "Green synthesis of silver nanoparticles using azadirachta indica aqueous leaf extract," Journal of Radiation Research and Applied Sciences, vol. 9, no. 1, pp. 1-7, 2016.

[42] T. Das, A. Pramanik, and D. Haldar, "On-line ammonia sensor and invisible security ink by fluorescent zwitterionic spirocyclic meisenheimer complex," Scientific Reports, vol. 7, no. 1, pp. 1-12, 2017.

[43] E. Alzahrani, "Chitosan membrane embedded with $\mathrm{ZnO} / \mathrm{CuO}$ nanocomposites for the photodegradation of fast green dye under artificial and solar irradiation," Analytical Chemistry Insights, vol. 13, Article ID 1177390118763361, 2018.

[44] A. Hebeish, A. El-Shafei, S. Sharaf, and S. Zaghloul, "Novel precursors for green synthesis and application of silver nanoparticles in the realm of cotton finishing," Carbohydrate Polymers, vol. 84, no. 1, pp. 605-613, 2011.

[45] L. Jeong and W. H. Park, "Preparation and characterization of gelatin nanofibers containing silver nanoparticles," International Journal of Molecular Sciences, vol. 15, no. 4, pp. 6857-6879, 2014.

[46] M. Rele, S. Kapoor, G. Sharma, and T. Mukherjee, "Reduction and aggregation of silver and thallium ions in viscous media," Physical Chemistry Chemical Physics, vol. 6, no. 3, pp. 590595, 2004.

[47] M. Kooti, S. Saiahi, and H. Motamedi, "Fabrication of silvercoated cobalt ferrite nanocomposite and the study of its antibacterial activity," Journal of Magnetism and Magnetic Materials, vol. 333, pp. 138-143, 2013.

[48] M. Vijayakumar, K. Priya, F. T. Nancy, A. Noorlidah, and A. B. A. Ahmed, "Biosynthesis, characterisation and antibacterial effect of plant-mediated silver nanoparticles using Artemisia nilagirica," Industrial Crops and Products, vol. 41, pp. 235-240, 2013.

[49] M. E. Khan, A. Mohammad, and M. H. Cho, "Nanoparticles based surface plasmon enhanced photocatalysis," in Green Photocatalysts, pp. 133-143, Springer, Berlin, Germany, 2020.

[50] E. Alzahrani, "Colorimetric detection based on localized surface plasmon resonance optical characteristics for sensing of mercury using green-synthesized silver nanoparticles," Journal of Analytical Methods in Chemistry, vol. 2020, Article ID 6026312, , 2020.

[51] E. Alzahrani, "Eco-friendly production of silver nanoparticles from peel of tangerine for degradation of dye," World Journal of Nano Science and Engineering, vol. 5, no. 1, pp. 10-16, 2015.

[52] E. Alzahrani, "Colorimetric detection based on localised surface plasmon resonance optical characteristics for the detection of hydrogen peroxide using acacia gum-stabilised silver nanoparticles," Analytical Chemistry Insights, vol. 12, Article ID 1177390116684686, 2017.

[53] E. Alzahrani and K. Welham, "Optimization preparation of the biosynthesis of silver nanoparticles using watermelon and study of its antibacterial activity," International Journal of Basic and Applied Sciences, vol. 3, no. 4, 2014.

[54] I. H. Chowdhury, S. Ghosh, R. Mouni, and M. Kanti Naskar, "Green synthesis of water-dispersible silver nanoparticles at room temperature using green carambola (star fruit) extract," Journal of Sol-Gel Science and Technology, vol. 73, no. 1, pp. 199-207, 2015.

[55] S. P. Chandran, M. Chaudhary, R. Pasricha, A. Ahmad, and M. Sastry, "Synthesis of gold nanotriangles and silver nanoparticles using aloevera plant extract," Biotechnology Progress, vol. 22, no. 2, pp. 577-583, 2006.

[56] P. Magudapathy, P. Gangopadhyay, B. K. Panigrahi, K. G. M. Nair, and S. Dhara, "Electrical transport studies of Ag nanoclusters embedded in glass matrix," Physica B: Condensed Matter, vol. 299, no. 1-2, pp. 142-146, 2001.

[57] A. Gomathi, S. R. Xavier Rajarathinam, A. Mohammed Sadiq, and S. Rajeshkumar, "Anticancer activity of silver nanoparticles synthesized using aqueous fruit shell extract of Tamarindus indica on MCF-7 human breast cancer cell line," Journal of Drug Delivery Science and Technology, vol. 55, Article ID 101376, 2020.

[58] S. S. Shankar, A. Rai, A. Ahmad, and M. Sastry, "Rapid synthesis of $\mathrm{Au}, \mathrm{Ag}$, and bimetallic Au core-Ag shell nanoparticles using neem (Azadirachta indica) leaf broth," Journal of Colloid and Interface Science, vol. 275, no. 2, pp. 496-502, 2004.

[59] M. Ismail, M. I. Khan, K. Akhtar et al., "Biosynthesis of silver nanoparticles: a colorimetric optical sensor for detection of hexavalent chromium and ammonia in aqueous solution," Physica E: Low-Dimensional Systems and Nanostructures, vol. 103, pp. 367-376, 2018.

[60] H. Bar, D. K. Bhui, G. P. Sahoo et al., "Green synthesis of silver nanoparticles using latex of Jatropha curcas," Colloids and Surfaces A: Physicochemical and Engineering Aspects, vol. 339, no. 1-3, pp. 134-139, 2009.

[61] D. R. Raj, S. Prasanth, T. V. Vineeshkumar, and C. Sudarsanakumar, "Ammonia sensing properties of tapered plastic optical fiber coated with silver nanoparticles/PVP/ PVA hybrid," Optics Communications, vol. 340, pp. 86-92, 2015.

[62] S. Kaviya, J. Santhanalakshmi, B. Viswanathan, J. Muthumary, and K. Srinivasan, "Biosynthesis of silver nanoparticles using citrus sinensis peel extract and its antibacterial activity," Spectrochimica Acta Part A: Molecular and Biomolecular Spectroscopy, vol. 79, no. 3, pp. 594-598, 2011.

[63] S. Kannaiyan and A. Gopal, "Biogenic synthesized silver colloid for colorimetric sensing of dichromate ion and antidiabetic studies," Research on Chemical Intermediates, vol. 43, no. 5, pp. 2693-2706, 2017.

[64] B. Onida, S. Fiorilli, A. Borello, G. Viscardi, D. A. McQuarrie, and E. Garrone, "Mechanism of the optical response of mesoporous silica impregnated with Reichardt's dye to $\mathrm{NH}_{3}$ and other gases," The Journal of Physical Chemistry B, vol. 108, no. 43, pp. 16617-16620, 2004.

[65] H. S. Mader and O. S. Wolfbeis, "Optical ammonia sensor based on upconverting luminescent nanoparticles," Analytical Chemistry, vol. 82, no. 12, pp. 5002-5004, 2010.

[66] M. K. Hedayati, F. Faupel, and M. Elbahri, "Review of plasmonic nanocomposite metamaterial absorber," Materials, vol. 7, no. 2, pp. 1221-1248, 2014.

[67] Z. Khan, J. Hussain, S. Kumar, A. A. Hashmi, and M. A. Malik, "Silver nanoparticles: green route, stability and effect of additives," Journal of Biomaterials and Nanobiotechnology, vol. 2, no. 4, p. 390, 2011.

[68] A. Amirjani and D. H. Fatmehsari, "Colorimetric detection of ammonia using smartphones based on localized surface plasmon resonance of silver nanoparticles," Talanta, vol. 176, pp. 242-246, 2018. 